\title{
Classifying Divergences in Cross-lingual AMR Pairs
}

\author{
Shira Wein Nathan Schneider \\ Georgetown University \\ \{sw1158, nathan.schneider\}@georgetown.edu
}

\begin{abstract}
Translation divergences are varied and widespread, challenging approaches that rely on parallel text. To annotate translation divergences, we propose a schema grounded in the Abstract Meaning Representation (AMR), a sentence-level semantic framework instantiated for a number of languages. By comparing parallel AMR graphs, we can identify specific points of divergence. Each divergence is labeled with both a type and a cause. We release a small corpus of annotated English-Spanish data, and analyze the annotations in our corpus.
\end{abstract}

\section{Introduction}

A variety of factors come into play in translating from one language into another (Dorr, 1990; Dorr and Voss, 1993). The resulting parallel texts are not always completely equivalent in meaning. Differences, or divergences, between the source and target may reflect lexical or grammatical differences between the two languages, stylistic considerations, or simply the translator's preference for idiomatic phrasing.

Identifying translation divergences may enable more nuanced use of parallel text in applications; for example, it has been shown that translation divergences have a measurable impact on machine translation (Vyas et al., 2018). This information on the types and causes of divergence enables these parallel texts to be more fully utilized in crosslingual natural language processing tasks. Specifically, different types of semantic divergences impact the performance of neural machine translation systems differently (Briakou and Carpuat, 2021), which motivates work to categorize and describe divergences in parallel texts.

Taking advantage of the fact that Abstract Meaning Representation (AMR) (Banarescu et al., 2013, 2019) resources and tools have been developed for a variety of languages $(\$ 2.3)$ and given the usefulness of AMR for downstream multilingual tasks such as machine translation (Song et al., 2019; Nguyen et al., 2021), we signal the need for categorization and detection of divergences in crosslingual AMR pairs.

The structure of an AMR reflects the semantic relations of the sentence, so structural divergences (a difference in the structure of an AMR graph, whether it be the label or role) between multilingual AMR pairs serve as a reflection of semantic differences between a sentence and its translation.

We develop and present a categorization schema to identify both the type and the cause of the divergence as being due to semantic divergence, annotation divergence, or syntactic divergence; this schema annotates both the type and cause of a structural divergence, to (1) make the data more adaptable to cross-lingual NLP applications, (2) identify non-literal translations, (3) make AMR more crosslinguistically consistent (Song et al., 2019), and (4) investigate the ways in which annotation, semantics, and syntax play a role in cross-lingual AMR parsing (Damonte, 2019).

We then annotate a set of 50 parallel EnglishSpanish AMRs annotations from The Little Prince (Migueles-Abraira, 2017) using our divergence schema and make these annotations available online. Using this small set of gold annotated data, we are able to explore the comprehensiveness and meaningfulness of this annotation schema.

Our contributions include:

- A novel annotation schema for the classification of semantic and translation divergences between cross-lingual pairs of AMRs (\$3)

- A small corpus of English-Spanish parallel AMRs annotated with our schema (\$4)

- An analysis of the ways in which crosslingual parallel AMRs may differ in annotation, which poses a problem for cross-lingual AMR parsing ( $\$ 4.3)$

We release the dataset for this work to enable 
cross-lingual Spanish-English analysis of AMR pairs, as well as promote expansion of this work to other languages. ${ }^{1}$

\section{Background and Related Work}

\subsection{Semantic Divergences}

Translation divergences occur when translation from one language to another results in a different meaning or structure (Dorr, 1994). These translation divergences can appear due to translation choices or to syntactic differences between the languages (Dorr, 1990; Dorr and Voss, 1993). The implications of these translation divergences include difficulties when using parallel texts for downstream tasks, because it can be difficult to identify why or how parallel sentences differ.

For example, a parallel corpus, such as a work of fiction, likely contains some non-literal translations. When training a machine translation system on this parallel corpus, these divergences present a problem if looking to produce as literal a translation as possible. An example of this from our corpus is shown in figure 1. The English sentence "Which is your planet?" is aligned to “¿De qué planeta eres?" which literally translates to "What planet are you from?" The Spanish sentence is less awkward than the original English sentence, and more explicitly asks about planet of origin, as opposed to ownership of a planet. This is a semantic divergence (due to translation choice), resulting in the Spanish AMR having a different focus (root).

Divergences have been explored with respect to synonymy (Gaillard et al., 2010) and diachronically (Montariol and Allauzen, 2021).

Other approaches have addressed whether and how given sentence pairs diverge. Carpuat et al. (2017) identify divergences in parallel corpora using a cross-lingual textual entailment system to identify less equivalent sentence pairs. Relatedly, work has been done to identify semantic divergences in parallel texts, classifying sentences as being divergent or non-divergent (Vyas et al., 2018).

Prior work which both categorically annotated and modeled semantic divergences includes the REFreSD dataset of English-French sentence pairs annotated with three types of divergences (subtree deletion, phrase replacement, and lexical substitution) based on a tree model (Briakou and Carpuat,

\footnotetext{
${ }^{1}$ The dataset for this work can be found at: https:// github.com/shirawein/spanish-english-amr-corpus
}

2020). Similarly, an attempt to classify these divergences automatically includes the hierarchical alignment scheme of Chinese and English parse trees which enables the identification and quantification of translation divergences (Deng and Xue, 2017).

These divergences have also been considered for downstream tasks. Prior work includes fine-tuning approach to account for non-literal translations in the pre-training of cross-lingual language models (Zhai et al., 2020).

\subsection{AMR for Cross-linguistic Divergences}

The Abstract Meaning Representation (AMR) is a framework for expressing the meaning of a whole sentence as a semantic graph (Banarescu et al., 2013, 2019). The semantically-oriented AMR framework has been extended to and studied in multiple languages, as well as cross-linguistically. However, it has not been used to formalize different kinds of translation divergences in cross-lingual parallel texts. We explore the use of Abstract Meaning Representations in formalizing the study of semantic translation divergences across parallel texts.

Abstract Meaning Representations (AMRs) are a type of semantic representation designed to pair a whole sentence with its meaning. AMRs are rooted, labeled graphs where each node is an instance of a semantic unit. The general purpose of the semantic representation is to capture the core logical elements of meaning. Annotation of AMR is lightweight as it does not represent morphology, articles, or tense, but does require a fair amount of training. Inter-annotator agreement is measured using Smatch, which calculates semantic overlap

English: Which is your planet?

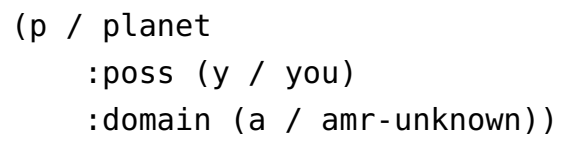

Spanish: ¿ De qué planeta eres ?

Literal translation: What planet are you from?

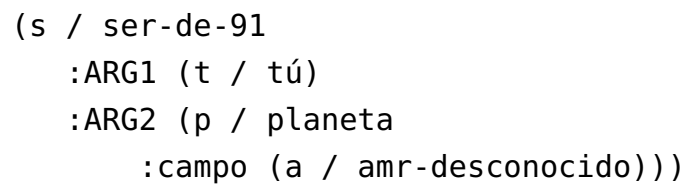

Figure 1: Example of a focus,sem divergence. 
between two AMRs (Cai and Knight, 2013). The root of the AMR is the focus, an attribute marked with : argN is a core argument role (where $N$ is some number $\geq 0$ ), and any other attribute (e.g., :opN, : domain, :manner) is a non-core role.

\subsection{Use of AMR in Many Languages and Cross-linguistically}

Abstract Meaning Representation is not an interlingua, but has since been studied in multiple languages and cross-linguistically (Xue et al., 2014; Li et al., 2016; Migueles-Abraira, 2017; Linh and Nguyen, 2019; Sobrevilla Cabezudo and Pardo, 2019; Choe et al., 2019).

AMR annotations of The Little Prince have been presented in English, Chinese ( $\mathrm{Li}$ et al., 2016), Spanish (Migueles-Abraira, 2017), and Vietnamese (Linh and Nguyen, 2019). Additional work has been done to adapt AMR annotation (Sobrevilla Cabezudo and Pardo, 2019) and alignment (Anchiêta and Pardo, 2020) to Portuguese, and Korean (Choe et al., 2019).

Cross-lingual studies of AMR primarily compare the structures between two AMRs representative of a parallel text, which may partially be due to the fact that AMR is not designed to be interlingual (Xue et al., 2014). AMRICA visualizes and automatically aligns AMRs, including two AMRs of a sentence and its translation, designed to facilitate research into cross-lingual AMRs (Saphra and Lopez, 2015). We take this as inspiration to use AMR pairs as a starting point for divergence classification between parallel texts.

Cross-lingual approaches to AMR parsing explore transfer learning techniques to generate parallel AMR annotations in multiple languages, and suggest that AMR can serve as a cross-lingual semantic representation capable of overcoming linguistic differences (Damonte and Cohen, 2018; Zhu et al., 2019; Blloshmi et al., 2020).

Additional work has explored whether structural differences across cross-lingual Chinese/English and English/Czech AMR pairs are due to syntactic idiosyncrasies (Xue et al., 2014), which can be of use in machine translation (Song et al., 2019; Nguyen et al., 2021).

\section{Cross-lingual AMR Divergence Annotation Schema}

We develop a categorization schema to be able to identify with granularity the type of structural

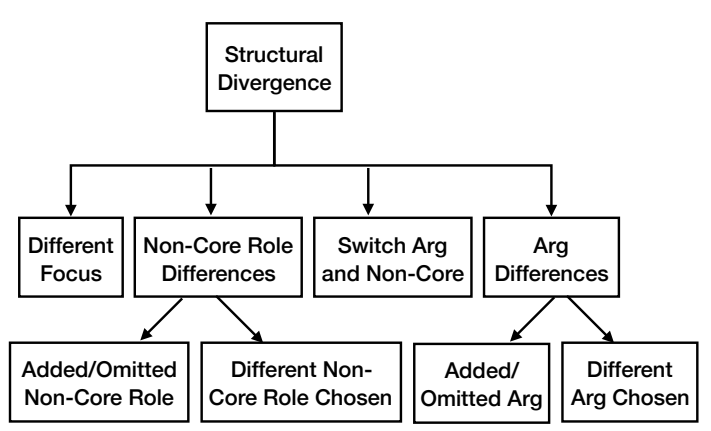

Figure 2: Types of structural divergence.

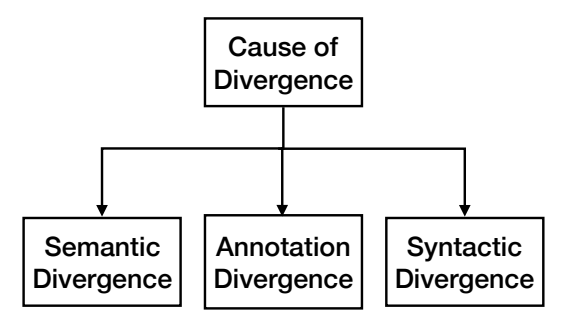

Figure 3: Causes of structural divergence.

divergence as well as the cause of the divergence between the two AMRs. Next, we annotate 50 Spanish-English AMR pairs using this schema.

If an AMR pair has a structural divergence, meaning there is some difference in the way that the two AMRs are structured, there must be both a type of divergence (figure 2) and a cause for the divergence (figure 3 ).

\subsection{Cause of Divergence}

The cause of the divergence can be:

- semantic divergences ("sem," due to translation choice)

- annotation divergences ("anno," due to annotation choice)

- or, syntactic divergences ("synt," inherent differences between the languages)

In semantic divergences, the Spanish translation is an inexact, non-literal, translation of the English sentence. For annotation divergences, the Spanish translation is a literal translation of the English sentence but the AMR was annotated differently nonetheless. Syntactic divergences arise because of some feature of either English or Spanish, the Spanish translation of the English sentences diverges in some way causing a structural divergence in the AMR.

\subsection{Type of Divergence}

As shown in figure 2, the type of structural divergence can be a:

1. different focus ("focus") 
2. a difference between the arguments / core roles ("diffarg" or "omitarg")

3. the same label/feature being an argument in one AMR and a non-core role in the other AMR ("switch")

4. a difference between non-core roles ("diffnoncore" or "omitnoncore")

These four subcategories are listed by decreasing degree of granularity and effect on the structure of the rest of the AMR.

If the focus is different between the two AMRs, then the entire rest of the structure differs by definition, because the arguments and non-core roles that the focus can take on differ. Therefore, if an AMR pair is annotated with each having a different focus, then it is not possible to then annotate argument differences or non-core role differences.

In the subsections that follow, we include and explain the annotation of 7 illustrative examples.

\subsection{Example of focus,sem}

One example of a focus difference due to translation, as described in $\S 2$, is shown in figure 1 . The focus, or root of the AMR graph, is the head/first node in the AMR. Here the focus differs because of the translation from the awkward "Which is your planet?" to the Spanish "¿De qué planeta eres?" which more explicitly asks about the geographic origin of the person being asked the question. The translation is a non-literal translation and the Spanish AMR reflects the Spanish sentence.

\subsection{Example of focus,anno}

An example of a different focus due to annotation choice includes the AMR pair for the English sentence Volcanic eruptions are like fires in a chimney . and the parallel Spanish sentence las erupciones volcánicas son como el fuego de una chimenea . The focus difference here, where in the English sentence the focus is erupt and in the Spanish sentence the focus is paracer $=$ seem, is due to annotation divergence in these gold annotations. The Spanish translation is a very literal translation of the English sentence. There are no other inherent language differences that place more emphasis on the "seeming" in the Spanish sentence either. Therefore, the difference is due to neither semantic divergence, being a literal translation, nor due to syntactic divergence; the difference is due to annotator discrepancy.

English: Volcanic eruptions are like fires in a chimney .

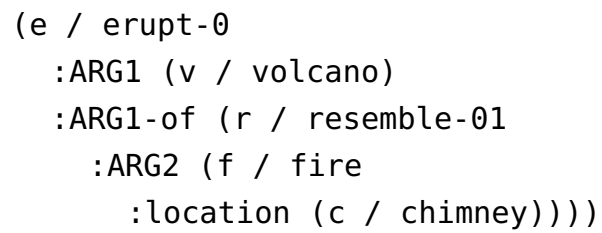

Spanish: Las erupciones volcánicas son como el fuego de una chimenea .

Literal translation: Volcanic eruptions are like the fire of a chimney.

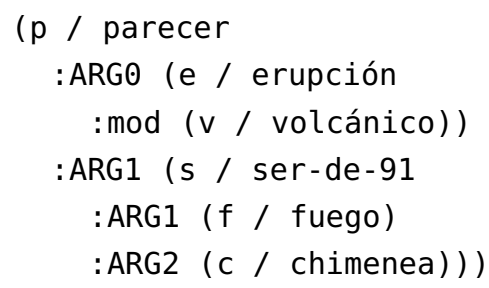

Similarly, if an argument is added/omitted or the label for an argument and non-core role are switched, then it is not possible to annotate a noncore role difference. It is still possible to have more than one structural divergence in an AMR pair because there are often multiple arguments and non-core roles in an AMR, so multiple differences may occur which do not explicitly encompass the others (as in §3.8).

\subsection{Example of switch,anno}

An AMR pair with English sentence: To forget $a$ friend is sad. and Spanish sentence Olvidar a un amigo es triste . is an example of the infrequent "switch." This pair annotates forget in English as an : ARGO, while olvidar = forget in Spanish is annotated as :domain. This is due to an annotation divergence. The English PropBank entry for sad02 denotes : ARGO as the being the causer of the sadness which makes it an appropriate choice for the English, and the same guidelines were being referenced for the Spanish AMR annotation. Therefore this is due to annotation divergence, and likely is an annotation error in the Spanish AMR.

Additionally, an argument difference can be due to adding/omitting an argument, or because for the same argument label (e.g. : argo) different arguments are chosen. If the same part of the sentence / feature of the AMR is featured as an :arge in one AMR and an : argl in the other AMR, this counts as two added/omitted arguments because the annotator judged that there was sufficient evidence for an : arge, but this was omitted in the other AMR, 
and vice versa.

\subsection{Example of diffarg,synt}

English: The fourth planet belonged to a businessman .

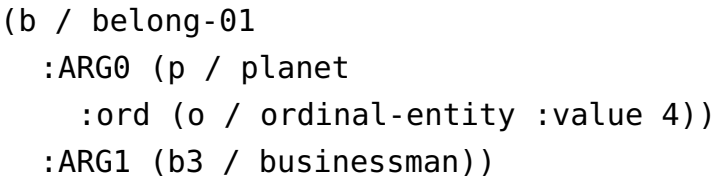

Spanish: El cuarto planeta era de un hombre de negocios .

Literal translation: The fourth planet was of a businessman.

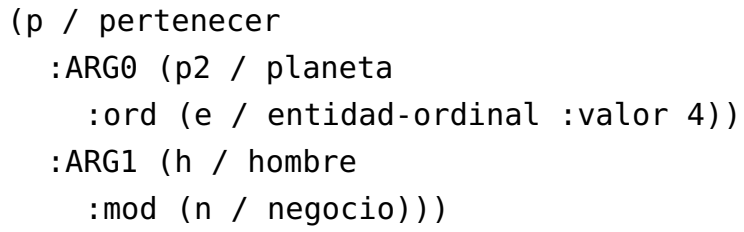

Here the :ARG1, businessman in English and hombre = man in Spanish, is due to syntactic divergence. Hombre de negocios is the Spanish translation of businessman, and literally means "man of business." Therefore it is structured in the AMR as hombre = man, $: \bmod$ negocio = business .

Similarly, a non-core role difference can be due to adding/omitting a non-core role, or because for the same argument label difference non-core roles are chosen.

\subsection{Example of diffnoncore,sem}

English: "I think it is time for breakfast, " she added an instant later .

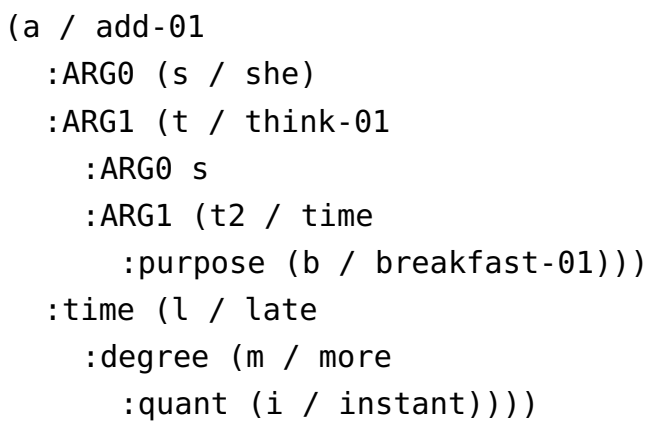

Spanish: "Creo que es la hora de desayunar" , añadió ella al instante .

Literal translation: "I think it's time for breakfast," she added instantly.

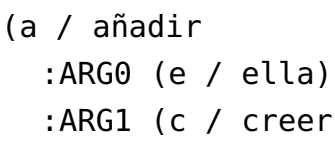

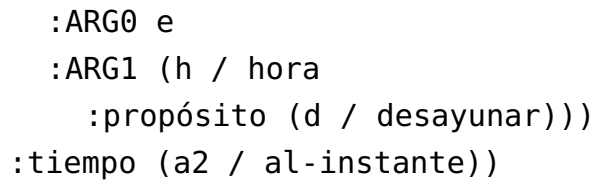

This difference in the non-core attribute (:time / : tiempo) is due to semantic divergences, being a non-literal Spanish translation. The English sentence says "an instant later," while the Spanish translation literally says "instantly." Therefore, the English AMR reflects the time as being an instant later and the Spanish AMR reflects the time as being at that instant.

\subsection{Example of omitnoncore,synt omitnoncore,synt (2)}

English: That is funny!

(f2 / funny :domain (t2 / that))

Spanish: ; Qué gracioso ! Literal translation: How funny!

(g / gracioso : grado ( $t / \tan )$ )

: grado corresponds to the English : degree, so the degree is included in the Spanish sentence, but the domain is included in the English sentence. This is due to syntactic divergence because a natural Spanish translation of "that is funny" would literally translate in English to "how funny," making degree more appropriate than domain.

\subsection{Example of no divergence}

English: Draw me a sheep ...

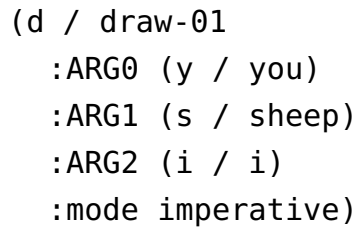

Spanish: Dibújame una oveja ... Literal translation: Draw me a sheep ...

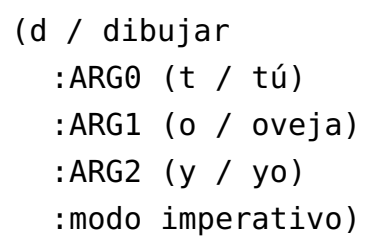

These AMRs are equivalent in every way, having equivalent sets of labels / relations, as well as the same arguments for each label. 


\section{Annotated Corpus}

In order to demonstrate the scope, effectiveness and use of this annotation schema, we decide to annotate a small corpus of English-Spanish sentences within the domain of literary works. We use a set of 50 English-Spanish AMR pairs, representing parallel sentences from The Little Prince (Migueles-Abraira et al., 2018). The work from which these 50 English-Spanish AMR pairs are derived also presents relevant linguistic issues hindering Spanish AMR annotation: NP ellipses, third person possessive pronouns, third person clitic pronouns, and the usage of se (Migueles-Abraira et al., 2018).

We annotated the 50 Spanish-English AMR pairs with a divergence classification, being empty if there is no structural divergence, or with at least one type,cause pair. It is possible to have more than one structural divergence so any structural divergence is listed with its cause.

Figure 5 shows the breakdown of cause of divergence for the 50 pairs in our corpus, as well as the relationship between type of divergence and cause. While different focus and different arg chosen are regularly due to annotation choice, different noncore role chosen or omitted non-core role are much more often due to translation choice.

\subsection{The Annotation Process}

All sentence pairs were annotated by the designer of the annotation scheme, who is fluent in both English and Spanish. There were few apparent difficulties in applying the scheme. The primary question was whether divergences that seemed like they were due to syntactic divergences were in fact inherent properties of the language, because this relies on expert knowledge of both languages. Ultimately this did not pose an issue because each of the three causes of structural divergence are mutually exclusive, so confirming that the property in question is in fact a feature inherent to the language can be validated by ensuring that there is not a difference in translation or annotation. The examples of language difference in $\$ 3.6$ and $\$ 3.8$ include justification for two instances of this classification. Reliability of the annotations could be validated by extending the study to include additional annotators and calculating inter-annotator agreement.

\subsection{Distribution of Corpus}

Of the 50 multilingual AMR pairs, 13 pairs had no structural divergence, 11 pairs had multiple divergences ( 9 pairs with 2 divergences and 2 pairs with 3 divergences), and 26 pairs have one structural divergence. There were 49 divergences in total.

Figure 5 shows the number of instances of every structural divergence caused by each of the three causes, with the types of structural divergences in rows and the causes of structural divergences in columns. Related information can also be seen in the form of a bar chart (Figure 4), which shows the proportion of instances for which a structural divergence is due to each cause.

Figures 5 and 4 show that there is a considerable discrepancy between type of divergence and cause. While non-core role divergences tend to be primarily due to semantic divergences, argument divergences tend to be due to annotation divergences or syntactic divergences. Change in focus tends to be due to an annotation divergence. The root serves as a representation of the central focus or topic of the sentence, so this relationship suggests that the central topic of the sentence is not changing between the parallel texts and instead the different focus annotation is due to annotator discrepancy.

\subsection{Implications for AMR Parsing \& Cross-lingual Tasks}

The results of this study indicate that cross-lingual AMR pairs actually do sometimes inherently differ because of properties of the language, so these differences need to be accounted for when developing cross-lingual AMR systems.

As (Xue et al., 2014) identifies divergences between Czech, Chinese, and English annotations in parallel texts which affect the degree to which AMR is an interlingua, we similarly investigate the causes and ways in which AMR is an interlingua, tested on the cross-lingual case of Spanish and English. Notably, due to the granularity of our annotation schema, we are able to both describe and quantify the divergence. We identify pairs of AMRs as being more than solely divergent, by rigorously classifying each type of divergence, as well as attributing each divergence to one of three causes: semantics, annotation, or syntax.

Additionally, we show that the structure provided in AMR allows many divergences to be identified and categorized by an annotator, even crosslingually. This motivates future work leveraging 


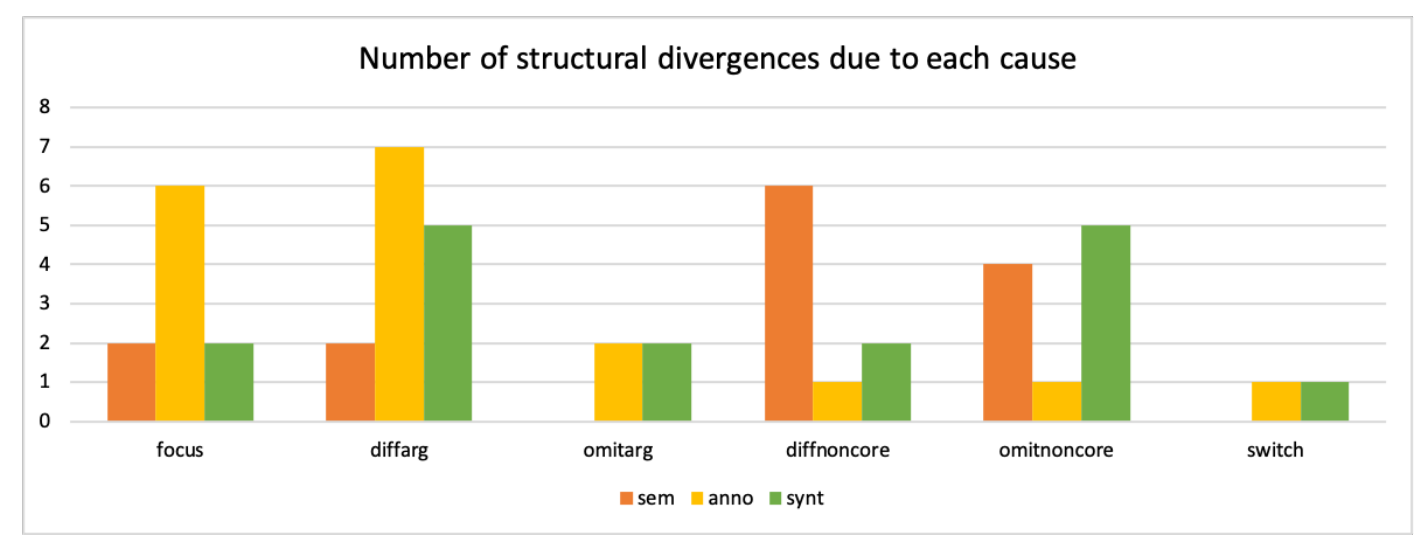

Figure 4: Bar chart reflecting the percent of instances for which each structural divergence was semantic, annotation, or syntactic.

\begin{tabular}{|l|r|r|r|r|}
\cline { 2 - 5 } \multicolumn{1}{c|}{} & sem & anno & synt & \\
\hline focus & 2 & 6 & 2 & 10 \\
\hline diffarg & 2 & 7 & 5 & 14 \\
\hline omitarg & 0 & 2 & 2 & 4 \\
\hline diffnoncore & 6 & 1 & 2 & 9 \\
\hline omitnoncore & 4 & 1 & 5 & 10 \\
\hline switch & 0 & 1 & 1 & 2 \\
\hline & 14 & 18 & 17 &
\end{tabular}

Figure 5: Number of instances of each structural divergence, and the number of times they were due to each cause of divergence. Greener cells indicate higher numbers and redder cells indicate lower numbers of instances. Sums across rows (types of divergence) and columns (causes of divergence) are found in clear cells to the right and bottom, respectively.

the semantic information captured by cross-lingual AMR pairs to identify sentences as being divergent or equivalent.

\section{Related Work on Cross-lingual Meaning Representations}

Related work has been done on cross-lingual adaptations of other meaning representations, including the development and analysis of cross-lingual semantic annotation schemes (Van Gysel et al., 2019). The Universal Dependencies framework has also been useful in the analysis of cross-lingual syntactic divergences (Nikolaev et al., 2020). Universal Conceptual Cognitive Annotation (UCCA) annotates grammatical meaning while abstracting away from the syntax of the language (Abend and Rappoport, 2013).

The Uniform Meaning Representation framework extends AMR with a focus on quantification and scope, as well as uniformity across languages (Van Gysel et al., 2021).

The Parallel Meaning Bank is a corpus of paral-

lel texts with corresponding linguistic annotations and Discourse Representation Structure annotations projected from English (Abzianidze et al., 2017). Prior work towards the production of parallel meaning banks focused on the alignment of informative translations-translations where more details are included in the target translation than the source text-through an inclusion relation symbol (Bos, 2014). Work on the Parallel Meaning Bank also includes a comparison of translations as being meaning-preserving or not, and these discrepancies as being largely due to: human annotation error, syntactic differences in definite articles, translation of proper names, or non-literal translations (van Noord et al., 2018).

Alignment for multilingual meaning representations has also been studied in relation to FrameNet, including recent work looking to unify a Multilingual FrameNet with alignments between all dozen FrameNet languages (Baker and Lorenzi, 2020).

The Prague Czech-English Dependency Treebank (PCEDT) similarly considers the adaptation of an English framework to a non-English extension, focusing on dependency annotation for the Czech translations of the Penn Treebank. PCEDT highlights some of the issues that arise out of automatically transferring annotation schema to another language. Ultimately, the authors find that the annotation schema are not sufficiently fine-grained to provide for a seamless conversion from annotation in one language to annotation in the other, and the difficulty of developing an annotation schema capable of this seamless transformation is unknown (Čmejrek et al., 2004).

$X L-A M R$ has used transfer learning to automatically produce AMR annotations for Chinese, German, Italian, and French, and provides qualitative 
analysis suggesting that even with limited training data, the parser is able to manage many structural divergences across languages (Blloshmi et al., 2020).

\section{Conclusion}

We have presented our annotation schema for classification of structural divergences in cross-lingual meaning representations and released 50 SpanishEnglish annotated examples. We demonstrate with our annotation schema and analysis of the annotated dataset that structural divergence in pairs of cross-lingual meaning representations can serve as a meaningful proxy of divergences between parallel texts. Therefore, tools which rely on highly literal translations, such as pre-trained machine translation systems, would benefit from applying this structural divergence annotation schema to crosslingual Abstract Meaning Representations of the data.

The scope of our empirical study was limited, however, because there are currently only 50 pairs of Spanish-English AMR annotations publicly available. Studying language pairs with more gold AMR data, or adapting the approach to alternate meaning representation formalisms, would further test the utility of our approach.

\section{Acknowledgments}

We thank reviewers for helpful feedback. This work is supported in part by a Clare Boothe Luce Scholarship.

\section{References}

Omri Abend and Ari Rappoport. 2013. UCCA: A semantics-based grammatical annotation scheme. In Proceedings of the 10th International Conference on Computational Semantics (IWCS 2013) Long Papers, pages 1-12, Potsdam, Germany. Association for Computational Linguistics.

Lasha Abzianidze, Johannes Bjerva, Kilian Evang, Hessel Haagsma, Rik van Noord, Pierre Ludmann, Duc-Duy Nguyen, and Johan Bos. 2017. The Parallel Meaning Bank: Towards a multilingual corpus of translations annotated with compositional meaning representations. In Proceedings of the 15th Conference of the European Chapter of the Association for Computational Linguistics: Volume 2, Short Papers, pages 242-247, Valencia, Spain. Association for Computational Linguistics.

Rafael Anchiêta and Thiago Pardo. 2020. Semantically inspired AMR alignment for the Portuguese language. In Proceedings of the 2020 Conference on Empirical Methods in Natural Language Processing (EMNLP), pages 1595-1600, Online. Association for Computational Linguistics.

Collin F. Baker and Arthur Lorenzi. 2020. Exploring crosslinguistic frame alignment. In Proceedings of the International FrameNet Workshop 2020: Towards a Global, Multilingual FrameNet, pages 77-84, Marseille, France. European Language Resources Association.

Laura Banarescu, Claire Bonial, Shu Cai, Madalina Georgescu, Kira Griffitt, Ulf Hermjakob, Kevin Knight, Philipp Koehn, Martha Palmer, and Nathan Schneider. 2013. Abstract Meaning Representation for sembanking. In Proceedings of the 7th Linguistic Annotation Workshop and Interoperability with Discourse, pages 178-186, Sofia, Bulgaria. Association for Computational Linguistics.

Laura Banarescu, Claire Bonial, Shu Cai, Madalina Georgescu, Kira Griffitt, Ulf Hermjakob, Kevin Knight, Philipp Koehn, Martha Palmer, and Nathan Schneider. 2019. Abstract Meaning Representation (AMR) 1.2.6 specification. https://github.com/ amrisi/amr-guidelines/blob/master/amr.md.

Rexhina Blloshmi, Rocco Tripodi, and Roberto Navigli. 2020. XL-AMR: Enabling cross-lingual AMR parsing with transfer learning techniques. In Proceedings of the 2020 Conference on Empirical Methods in Natural Language Processing (EMNLP), pages 2487-2500, Online. Association for Computational Linguistics.

Johan Bos. 2014. Semantic annotation issues in parallel meaning banking. In ACL 2014.

Eleftheria Briakou and Marine Carpuat. 2020. Detecting fine-grained cross-lingual semantic divergences without supervision by learning to rank. In Proceedings of the 2020 Conference on Empirical Methods in Natural Language Processing (EMNLP), pages 1563-1580, Online. Association for Computational Linguistics.

Eleftheria Briakou and Marine Carpuat. 2021. Beyond noise: Mitigating the impact of fine-grained semantic divergences on neural machine translation. CoRR, abs/2105.15087.

Shu Cai and Kevin Knight. 2013. Smatch: an evaluation metric for semantic feature structures. In Proceedings of the 51st Annual Meeting of the Association for Computational Linguistics (Volume 2: Short Papers), pages 748-752, Sofia, Bulgaria. Association for Computational Linguistics.

Marine Carpuat, Yogarshi Vyas, and Xing Niu. 2017. Detecting cross-lingual semantic divergence for neural machine translation. In Proceedings of the First Workshop on Neural Machine Translation, pages 6979, Vancouver. Association for Computational Linguistics. 
Hyonsu Choe, Jiyoon Han, Hyejin Park, and Hansaem Kim. 2019. Copula and case-stacking annotations for Korean AMR. In Proceedings of the First International Workshop on Designing Meaning Representations, pages 128-135, Florence, Italy. Association for Computational Linguistics.

Martin Čmejrek, Jan Cuřín, and Jiří Havelka. 2004. Prague Czech-English Dependency Treebank: Any hopes for a common annotation scheme? In Proceedings of the Workshop Frontiers in Corpus Annotation at HLT-NAACL 2004, pages 47-54, Boston, Massachusetts, USA. Association for Computational Linguistics.

Marco Damonte. 2019. Understanding and Generating Language with Abstract Meaning Representation. Ph.D. thesis, University of Edinburgh.

Marco Damonte and Shay B. Cohen. 2018. Crosslingual Abstract Meaning Representation parsing. In Proceedings of the 2018 Conference of the North American Chapter of the Association for Computational Linguistics: Human Language Technologies, Volume 1 (Long Papers), pages 1146-1155, New Orleans, Louisiana. Association for Computational Linguistics.

Dun Deng and Nianwen Xue. 2017. Translation divergences in Chinese-English machine translation: An empirical investigation. Computational Linguistics, 43(3):521-565.

Bonnie Dorr. 1990. Solving thematic divergences in machine translation. In Proceedings of the 28th Annual Meeting on Association for Computational Linguistics, ACL '90, page 127-134, USA. Association for Computational Linguistics.

Bonnie J. Dorr. 1994. Machine translation divergences: A formal description and proposed solution. Computational Linguistics, 20(4):597-633.

Bonnie J. Dorr and Clare R. Voss. 1993. Constraints on the space of MT divergences.

Benoît Gaillard, Yannick Chudy, Pierre Magistry, ShuKai Hsieh, and Emmanuel Navarro. 2010. Graph representation of synonymy and translation resources for crosslinguistic modelisation of meaning. In Proceedings of the 24th Pacific Asia Conference on Language, Information and Computation, pages 819-830, Tohoku University, Sendai, Japan. Institute of Digital Enhancement of Cognitive Processing, Waseda University.

Bin Li, Yuan Wen, Weiguang Qu, Lijun Bu, and Nianwen Xue. 2016. Annotating The Little Prince with Chinese AMRs. In Proceedings of the 10th Linguistic Annotation Workshop held in conjunction with ACL 2016 (LAW-X 2016), pages 7-15, Berlin, Germany. Association for Computational Linguistics.
Ha Linh and Huyen Nguyen. 2019. A case study on meaning representation for Vietnamese. In Proceedings of the First International Workshop on Designing Meaning Representations, pages 148-153, Florence, Italy. Association for Computational Linguistics.

Noelia Migueles-Abraira. 2017. A study towards Spanish Abstract Meaning Representation. Master's thesis, University of the Basque Country.

Noelia Migueles-Abraira, Rodrigo Agerri, and Arantza Diaz de Ilarraza. 2018. Annotating Abstract Meaning Representations for Spanish. In Proceedings of the Eleventh International Conference on Language Resources and Evaluation (LREC 2018), Miyazaki, Japan. European Language Resources Association (ELRA).

Syrielle Montariol and Alexandre Allauzen. 2021. Measure and evaluation of semantic divergence across two languages. In Proceedings of the 59th Annual Meeting of the Association for Computational Linguistics and the 11th International Joint Conference on Natural Language Processing (Volume 1: Long Papers), pages 1247-1258, Online. Association for Computational Linguistics.

Long HB Nguyen, Viet H Pham, and Dien Dinh. 2021. Improving neural machine translation with AMR semantic graphs. Mathematical Problems in Engineering, 2021.

Dmitry Nikolaev, Ofir Arviv, Taelin Karidi, Neta Kenneth, Veronika Mitnik, Lilja Maria Saeboe, and Omri Abend. 2020. Fine-grained analysis of crosslinguistic syntactic divergences. In Proceedings of the 58th Annual Meeting of the Association for Computational Linguistics, pages 1159-1176, Online. Association for Computational Linguistics.

Naomi Saphra and Adam Lopez. 2015. AMRICA: an AMR inspector for cross-language alignments. In Proceedings of the 2015 Conference of the North American Chapter of the Association for Computational Linguistics: Demonstrations, pages 36-40, Denver, Colorado. Association for Computational Linguistics.

Marco Antonio Sobrevilla Cabezudo and Thiago Pardo. 2019. Towards a general Abstract Meaning Representation corpus for Brazilian Portuguese. In Proceedings of the 13th Linguistic Annotation Workshop, pages 236-244, Florence, Italy. Association for Computational Linguistics.

Linfeng Song, Daniel Gildea, Yue Zhang, Zhiguo Wang, and Jinsong Su. 2019. Semantic neural machine translation using AMR. Transactions of the Association for Computational Linguistics, 7:19-31.

Jens E. L. Van Gysel, Meagan Vigus, Jayeol Chun, Kenneth Lai, Sarah Moeller, Jiarui Yao, Tim O'Gorman, Andrew Cowell, William Croft, Chu-Ren Huang, Jan Hajič, James H. Martin, Stephan Oepen, Martha 
Palmer, James Pustejovsky, Rosa Vallejos, and Nianwen Xue. 2021. Designing a Uniform Meaning Representation for natural language processing. $K I$ Künstliche Intelligenz.

Jens E. L. Van Gysel, Meagan Vigus, Pavlina Kalm, Sook-kyung Lee, Michael Regan, and William Croft. 2019. Cross-linguistic semantic annotation: Reconciling the language-specific and the universal. In Proceedings of the First International Workshop on Designing Meaning Representations, pages 1-14, Florence, Italy. Association for Computational Linguistics.

Rik van Noord, Lasha Abzianidze, Hessel Haagsma, and Johan Bos. 2018. Evaluating scoped meaning representations. In Proceedings of the Eleventh International Conference on Language Resources and Evaluation (LREC 2018), Miyazaki, Japan. European Language Resources Association (ELRA).

Yogarshi Vyas, Xing Niu, and Marine Carpuat. 2018. Identifying semantic divergences in parallel text without annotations. In Proceedings of the 2018 Conference of the North American Chapter of the Association for Computational Linguistics: Human Language Technologies, Volume 1 (Long Papers), pages 1503-1515, New Orleans, Louisiana. Association for Computational Linguistics.

Nianwen Xue, Ondřej Bojar, Jan Hajič, Martha Palmer, Zdeňka Urešová, and Xiuhong Zhang. 2014. Not an interlingua, but close: Comparison of English AMRs to Chinese and Czech. In Proceedings of the Ninth International Conference on Language Resources and Evaluation (LREC'14), pages 17651772, Reykjavik, Iceland. European Language Resources Association (ELRA).

Yuming Zhai, Gabriel Illouz, and Anne Vilnat. 2020. Detecting non-literal translations by fine-tuning cross-lingual pre-trained language models. In Proceedings of the 28th International Conference on Computational Linguistics, pages 5944-5956, Barcelona, Spain (Online). International Committee on Computational Linguistics.

Huaiyu Zhu, Yunyao Li, and Laura Chiticariu. 2019. Towards universal semantic representation. In Proceedings of the First International Workshop on Designing Meaning Representations, pages 177-181, Florence, Italy. Association for Computational Linguistics. 